\title{
The Interplay of Classes of Algorithmically Random Objects
}

\author{
QUINN CULVER \\ CHRISTOPHER P. PORTER
}

\begin{abstract}
We study algorithmically random closed subsets of $2^{\omega}$, algorithmically random continuous functions from $2^{\omega}$ to $2^{\omega}$, and algorithmically random Borel probability measures on $2^{\omega}$, especially the interplay between these three classes of objects. Our main tools are preservation of randomness and its converse, the no randomness ex nihilo principle, which say together that given an almost-everywhere defined computable map between an effectively compact probability space and an effective Polish space, a real is Martin-Löf random for the pushforward measure if and only if its preimage is random with respect to the measure on the domain. These tools allow us to prove new facts, some of which answer previously open questions, and reprove some known results more simply.

Our main results are the following. First we answer an open question in Barmpalias, Brodhead, Cenzer, Remmel, and Weber [3] by showing that $X \subseteq 2^{\omega}$ is a random closed set if and only if it is the set of zeros of a random continuous function on $2^{\omega}$. As a corollary we obtain the result that the collection of random continuous functions on $2^{\omega}$ is not closed under composition. Next, we construct a computable measure $Q$ on the space of measures on $2^{\omega}$ such that $X \subseteq 2^{\omega}$ is a random closed set if and only if $\mathcal{X}$ is the support of a $Q$-random measure. We also establish a correspondence between random closed sets and the random measures studied in Culver [8]. Lastly, we study the ranges of random continuous functions, showing that the Lebesgue measure of the range of a random continuous function is always contained in $(0,1)$.
\end{abstract}

2010 Mathematics Subject Classification 03D32 (primary); 03D80, 68Q30 (secondary)

Keywords: algorithmic randomness, computable analysis, random closed sets, random functions, random measures

\section{Introduction}

In this paper, we have two primary goals: (1) to study the interplay between algorithmically random closed sets on $2^{\omega}$, algorithmically random continuous functions on $2^{\omega}$, and 
algorithmically random measures on $2^{\omega}$; and (2) to apply two central results, namely the preservation of randomness principle and the no randomness ex nihilo principle, to the study of the algorithmically random objects listed above.

Barmpalias, Brodhead, Cenzer, Dashti and Weber initiated the study of algorithmically random closed subsets of $2^{\omega}$ in [2]. Algorithmically random closed sets were further studied in, for instance, Axon [1], Diamondstone and Kjos-Hanssen [9], and Cenzer and Weber [7]. In the spirit of the definition of an algorithmically random closed set, Barmpalias, Brodhead, Cenzer, Remmel and Weber defined a notion of algorithmically random continuous function on $2^{\omega}$ in [3]. The connection between random closed sets and effective capacities was explored in Brodhead, Cenzer, Toska, and Wyman [5]. More recently, Culver has studied algorithmically random measures on $2^{\omega}$ in Culver [8].

One of the central results in [3] is that the set of zeros of a random continuous function of $2^{\omega}$ is a random closed subset of $2^{\omega}$. Inspired by this result, we here investigate similar bridge results, which allow us to transfer information about one class of algorithmically random objects to another.

Two tools that are central to our investigation, mentioned in (2) above, are the preservation of randomness principle and the no randomness ex nihilo principle. In $2^{\omega}$, the space of infinite binary sequences, the preservation of randomness principle tells us that if $\Phi: 2^{\omega} \rightarrow 2^{\omega}$ is an effective map and $\mu$ is a computable measure on $2^{\omega}$, then $\Phi$ maps $\mu$-random members of $2^{\omega}$ to members of $2^{\omega}$ that are random with respect to the measure $\nu$ obtained by pushing $\mu$ forward via $\Phi$. Furthermore, the no randomness ex nihilo principle tells us that any sequence that is random with respect to $\nu$ is the image of some $\mu$-random sequence under $\Phi$. Used in tandem, these two principles allow us to conclude that the image of the $\mu$-random sequences under $\Phi$ are precisely the $\nu$-random sequences.

With the exception of [8], the studies listed above do not make use of these two tools used in tandem. As we will show, they not only allow for the simplification of a number of proofs in the above-listed studies, but they also allow us to answer a number of questions that were left open in these studies.

The outline of the remainder of this paper is as follows. In Section 2, we provide the requisite background for the rest of the paper. In Section 3, we review the basics of algorithmic randomness, including preservation and the no randomness ex nihilo principle. We also provide the definitions of algorithmic randomness for closed sets of $2^{\omega}$, random continuous functions on $2^{\omega}$, and measures on $2^{\omega}$ and list some basic properties of these objects. Section 4 contains simplified proofs of some previously 
obtained results from [2] and [3], as well as a proof of a conjecture in [3] that every random closed subset of $2^{\omega}$ is the set of zeros of a random continuous function on $2^{\omega}$. We study the support of a certain class of random measures in Section 5 and establish a correspondence between between random closed sets and the random measures studied in [8]. Lastly, in Section 6, we prove that the Lebesgue measure of the range of a random continuous function on $2^{\omega}$ is always non-zero, from which it follows that no random continuous function is injective. This yields a new proof of this previously established result (in [3]). We also strengthen a result in [3], namely, that not every random continuous function is surjective, by proving that no random continuous function is surjective, from which it follows that the Lebesgue measure of the range of a random continuous function is never equal to one.

The results in this paper can also be found in [8], which the reader can consult for additional background material.

\section{Background}

\subsection{Some topological and measure-theoretic basics}

For $n=\{0,1, \ldots n-1\} \in \omega$, the set of all finite strings over the alphabet $n$ is denoted $n^{<\omega}$. When $n=2$, we let $\sigma_{0}, \sigma_{1}, \sigma_{2}, \ldots$ be the canonical length-lexicographic enumeration of $2^{<\omega}$, so that $\sigma_{0}=\epsilon$ (the empty string), $\sigma_{1}=0, \sigma_{2}=1$, etc.

$n^{\omega}$ is the space of all infinite sequences over the alphabet $n$. The elements of $n^{\omega}$ are also called reals. The product topology on $n^{\omega}$ is generated by the clopen sets

$$
\llbracket \sigma \rrbracket=\left\{x \in n^{\omega}: x \succ \sigma\right\},
$$

where $\sigma \in n^{<\omega}$ and $x \succ \sigma$ means that $\sigma$ is an initial segment of $x$. When $x$ is a real and $k \in \omega, x\lceil k$ denotes the initial segment of $x$ of length $k$.

For $\sigma, \tau \in n^{<\omega}, \sigma^{\frown} \tau$ denotes the concatenation of $\sigma$ and $\tau$. In some cases, we will write this concatenation as $\sigma \tau$.

A tree is a subset of $n^{<\omega}$ that is closed under initial segments, ie, $T \subseteq n^{<\omega}$ is a tree if $\sigma \in T$ whenever $\tau \in T$ and $\sigma \preceq \tau$. A path through a tree $T \subseteq n^{<\omega}$ is a real $x \in n^{\omega}$ satisfying $x\lceil k \in T$ for every $k$. The set of all paths through a tree $T$ is denoted [T]. Recall the correspondence between closed sets and trees.

Proposition 2.1 A set $C \subseteq n^{\omega}$ is closed if and only if $C=[T]$ for some tree $T \subseteq n^{<\omega}$. Moreover, $C$ is non-empty if and only if $T$ is infinite. 
A measure $\mu$ on $n^{\omega}$ is a function that assigns to each Borel subset of $n^{\omega}$ a number in the unit interval $[0,1]$ and satisfies $\mu\left(\bigcup_{i \in \omega} B_{i}\right)=\sum_{i \in \omega} \mu\left(B_{i}\right)$ whenever the $B_{i}$ 's are pairwise disjoint. Carathéodory's extension theorem guarantees that the conditions

- $\mu(\llbracket \epsilon \rrbracket)=1$ and

- $\mu(\llbracket \sigma \rrbracket)=\mu(\llbracket \sigma 0 \rrbracket)+\mu(\llbracket \sigma 1 \rrbracket)+\ldots+\mu(\llbracket \sigma \frown(n-1) \rrbracket)$ for all $\sigma \in n^{<\omega}$

uniquely determine a measure on $n^{\omega}$. We thus identify a measure with a function $\mu: n^{<\omega} \rightarrow[0,1]$ satisfying the above conditions and $\mu(\sigma)$ is often written instead of $\mu(\llbracket \sigma \rrbracket)$. The Lebesgue measure $\lambda$ on $n^{\omega}$ is defined by $\lambda(\sigma)=n^{-|\sigma|}$ for each string $\sigma \in n^{<\omega}$.

Given a measure $\mu$ on $n^{\omega}$ and $\sigma, \tau \in n^{<\omega}, \mu(\sigma \tau \mid \sigma)$ is defined to be

$$
\mu(\sigma \tau \mid \sigma)=\frac{\mu(\llbracket \sigma \tau \rrbracket)}{\mu(\llbracket \sigma \rrbracket)} .
$$

\subsection{Some computability theory}

We assume the reader is familiar with the basic concepts of computability theory as found, for instance, in the early chapters of Soare [14].

A $\Sigma_{1}^{0}$ class $S \subseteq n^{\omega}$ is an effectively open set, ie, an effective union of basic clopen subsets of $n^{\omega} . P \subseteq n^{\omega}$ is a $\Pi_{1}^{0}$ class if $2^{\omega} \backslash P$ is a $\Sigma_{1}^{0}$ class.

A partial function $\Phi: \subseteq n^{\omega} \rightarrow m^{\omega}$ is computable if the preimage of a $\Sigma_{1}^{0}$ subset of $m^{\omega}$ is a $\Sigma_{1}^{0}$ subset of the domain of $\Phi$, uniformly; that is, if for every $\Sigma_{1}^{0}$ class $U \subseteq m^{\omega}$, there is a $\Sigma_{1}^{0}$ class $V \subseteq n^{\omega}$ such that $\Phi^{-1}(U)=V \cap \operatorname{dom}(\Phi)$, and an index for $V$ can be uniformly computed from an index for $U$. Equivalently, $\Phi: \subseteq n^{\omega} \rightarrow m^{\omega}$ is computable if there is an oracle Turing machine that when given $x \in n^{\omega}$ (as an oracle) and $k \in \omega$ outputs $\Phi(x)(k)$. We can relativize the notion of a computable function $\Phi: \subseteq n^{\omega} \rightarrow m^{\omega}$ to any oracle $z \in 2^{\omega}$ to obtain a $z$-computable function.

A measure $\mu$ on $n^{\omega}$ is computable if $\mu(\sigma)$ is a computable real number, uniformly in $\sigma \in n^{<\omega}$. Clearly, the Lebesgue measure $\lambda$ is computable.

If $\mu$ is a computable measure on $n^{\omega}$ and $\Phi: \subseteq n^{\omega} \rightarrow m^{\omega}$ is a computable function defined on a set of $\mu$-measure one, then the pushforward measure $\mu_{\Phi}$ defined by

$$
\mu_{\Phi}(\sigma)=\mu\left(\Phi^{-1}(\sigma)\right)
$$

for each $\sigma \in m^{<\omega}$ is a computable measure. 


\section{Algorithmically random objects}

In this section, we lay out the definitions of the various algorithmically random objects that are the subject of this study. For more details, see Nies [12] or Downey and Hirschfeldt [10].

\subsection{Algorithmically random sequences}

Definition 3.1 Let $\mu$ be a computable measure on $n^{\omega}$ and let $z \in m^{\omega}$.

(i) A $\mu$-Martin-Löf test relative to $z$ (or simply a $\mu$-test relative to $z$ ) is a uniformly $\Sigma_{1}^{0, z}$ sequence $\left(U_{i}\right)_{i \in \omega}$ of subsets of $n^{\omega}$ with $\mu\left(U_{n}\right) \leq 2^{-n}$.

(ii) $x \in n^{\omega}$ passes such a test $\left(U_{i}\right)_{i \in \omega}$ if $x \notin \bigcap_{n} U_{n}$.

(iii) $x \in n^{\omega}$ is $\mu$-Martin-Löf random relative to $z$ if $x$ passes every $\mu$-Martin-Löf test relative to $z$.

We will often write "random" instead of "Martin-Löf random," since we are only working with one notion of randomness in this paper (although there are other reasonable notions of algorithmic randomness that one might consider). The collection of $\mu$-random sequences relative to $z$ will be denoted $\operatorname{MLR}_{\mu}^{z}$. When $z$ is computable we simply write $\mathrm{MLR}_{\mu}$, say that $x$ is $\mu$-random, call $\left(U_{i}\right)_{i \in \omega}$ a $\mu$-test, etc.

The following is well-known and straightforward.

Proposition 3.2 Let $\mu$ be a computable measure on $n^{\omega}$ and $z \in m^{\omega}$. If $C \subseteq n^{\omega}$ is $\Pi_{1}^{0, z}$ and $\mu(C)=0$, then $C \cap \mathrm{MLR}_{\mu}^{z}=\emptyset$.

The following is likely folklore, but was at least observed in Bienvenu and Porter [4].

Proposition 3.3 Let $\mu$ be a computable measure on $n^{\omega}$. If $\Phi: \subseteq n^{\omega} \rightarrow m^{\omega}$ is computable with $\mu(\operatorname{dom}(\Phi))=1$, then $\operatorname{MLR}_{\mu} \subseteq \operatorname{dom}(\Phi)$.

The next result is also folklore; see, for instance, Cenzer and Remmel [6, Theorem 3.4.8(3)].

Lemma 3.4 (Folklore) Let $\Phi: \subseteq 2^{\omega} \rightarrow 2^{\omega}$ be computable and $C$ a $\Pi_{1}^{0}$ subset of $\operatorname{dom}(\Phi)$. Then $\Phi(C) \in \Pi_{1}^{0}$, uniformly.

One of the central tools that we will use in this study is the following. 
Theorem 3.5 (Preservation of Randomness (Zvonkin and Levin [16]) and No Randomness Ex Nihilo (Shen [13])) Let $\Phi: \subseteq 2^{\omega} \rightarrow 2^{\omega}$ be computable with $\lambda(\operatorname{dom}(\Phi))=1$.

(i) If $x \in \mathrm{MLR}_{\lambda}$ then $\Phi(x) \in \mathrm{MLR}_{\lambda_{\Phi}}$.

(ii) If $y \in \mathrm{MLR}_{\lambda_{\Phi}}$, then there is $x \in \operatorname{MLR}_{\lambda}$ such that $\Phi(x)=y$.

Proof (i) If $\Phi(x) \notin \mathrm{MLR}_{\lambda_{\Phi}}$, then $\Phi(x) \in \bigcap_{n} V_{n}$ for some $\lambda_{\Phi}$-test $\left(V_{i}\right)_{i \in \omega}$. But then $x \in \bigcap_{n} \Phi^{-1}\left(V_{n}\right)$ and $\lambda\left(\Phi^{-1}\left(V_{n}\right)\right) \leq 2^{-n}$. Moreover, because $\Phi$ is computable (on its domain), $\Phi^{-1}\left(V_{n}\right)=U_{n} \cap \operatorname{dom}(\Phi)$ for some $\Sigma_{1}^{0}$ class $U_{n}$. Since $\lambda(\operatorname{dom}(\Phi))=1$, $\lambda\left(U_{n}\right) \leq 2^{-n}$. Thus $x \notin \mathrm{MLR}_{\lambda}$.

(ii) Let $\left(U_{i}\right)_{i \in \omega}$ be a universal test for $\lambda$-randomness and set $K_{n}=2^{\omega} \backslash U_{n}$, where $K_{n} \subseteq \mathrm{MLR}_{\lambda} \subseteq \operatorname{dom}(\Phi)$ by Proposition 3.3. Then $\Phi\left(K_{n}\right)$ is uniformly $\Pi_{1}^{0}$ by Lemma 3.4, so $2^{\omega} \backslash \Phi\left(K_{n}\right)$ is uniformly $\Sigma_{1}^{0}$. Because

$$
\lambda_{\Phi}\left(2^{\omega} \backslash \Phi\left(K_{n}\right)\right)=1-\lambda_{\Phi}\left(\Phi\left(K_{n}\right)\right) \leq 1-\lambda\left(K_{n}\right) \leq 2^{-n},
$$

the sets $2^{\omega} \backslash \Phi\left(K_{n}\right)$ form a test for $\lambda_{\Phi}$-randomness. So if $y \in \mathrm{MLR}_{\lambda_{\Phi}}$, then $y \notin 2^{\omega} \backslash \Phi\left(K_{n}\right)$ for some $n$, ie, $y \in \Phi\left(K_{n}\right)$. The proof is now complete since $K_{n} \subseteq \mathrm{MLR}_{\lambda}$.

The above proof immediately generalizes to computable functions $\Phi: \subseteq n^{\omega} \rightarrow m^{\omega}$ satisfying $\lambda(\operatorname{dom}(\Phi))=1$. In what follows, we will occasionally use Theorem 3.5 for such functions. We will also use a relativization of Theorem 3.5.

Corollary 3.6 Let $\Phi: \subseteq 2^{\omega} \rightarrow 2^{\omega}$ be a function that is computable relative to $z \in 2^{\omega}$ and satisfies $\lambda(\operatorname{dom}(\Phi))=1$.

(i) If $x \in \mathrm{MLR}_{\lambda}^{z}$ then $\Phi(x) \in \mathrm{MLR}_{\lambda_{\Phi}}^{z}$.

(ii) If $y \in \operatorname{MLR}_{\lambda_{\Phi}}^{z}$, then there is $x \in \operatorname{MLR}_{\lambda}^{z}$ such that $\Phi(x)=y$.

Lastly, the following result, known as van Lambalgen's theorem, will be useful to us. Given a measure $\mu$ on $m^{\omega}$ and a measure $\nu$ on $n^{\omega}, \mu \otimes \nu$ denotes the product measure on $m^{\omega} \times n^{\omega}$.

Theorem 3.7 ([15]) Let $\mu$ and $\nu$ be computable measures on $m^{\omega}$ and $n^{\omega}$, respectively. Then for $(x, y) \in m^{\omega} \times n^{\omega},(x, y) \in \operatorname{MLR}_{\mu \otimes \nu}$ if and only if $x \in \operatorname{MLR}_{\mu}^{y}$ and $y \in \operatorname{MLR}_{\nu}$. 


\subsection{Algorithmically random closed subsets of $2^{\omega}$}

Let $\mathcal{C}\left(2^{\omega}\right)$ denote the collection of all non-empty closed subsets of $2^{\omega}$. As noted in Proposition 2.1, these are the sets of paths through infinite binary trees. Thus to randomly generate a non-empty closed set, it suffices to randomly generate an infinite tree. Following Barmpalias, Brodhead, Cenzer, Dashti, and Weber [2], we will code infinite trees by reals in $3^{\omega}$, thereby reducing the process of randomly generating infinite trees to the process of randomly generating reals.

Given $x \in 3^{\omega}$, define a tree $T_{x} \subseteq 2^{<\omega}$ inductively as follows. First $\epsilon$, the empty string is automatically in $T_{x}$. Now suppose $\sigma \in T_{x}$ is the $(i+1)$-st extendible node in $T_{x}$. Then

- $\sigma 0 \in T_{x}$ and $\sigma 1 \notin T_{x}$ if $x(i)=0$;

- $\sigma 0 \notin T_{x}$ and $\sigma 1 \in T_{x}$ if $x(i)=1$;

- $\sigma 0 \in T_{x}$ and $\sigma 1 \in T_{x}$ if $x(i)=2$.

Under this coding $T_{x}$ has no dead ends and hence is always infinite. Note that every tree without dead ends can be coded by some $x \in 2^{\omega}$.

Definition 3.8 A non-empty closed set $C \in \mathcal{C}\left(2^{\omega}\right)$ is a random closed set if $C=\left[T_{x}\right]$ for some $x \in M^{\prime} R_{\lambda}$.

The main facts about random closed sets that we will use in the sequel are as follows.

Theorem 3.9 ([2]) Every random closed set has Lebesgue measure zero.

Theorem 3.10 ([2]) Every random closed set is perfect.

\subsection{Algorithmically random continuous functions on $2^{\omega}$}

Let $\mathcal{F}\left(2^{\omega}\right)$ denote the collection of all continuous $F: \subseteq 2^{\omega} \rightarrow 2^{\omega}$. To define a random continuous function, we code each element of $\mathcal{F}\left(2^{\omega}\right)$ by a real $x \in 3^{\omega}$ (as carried out in Barmpalias, Brodhead, Cenzer, Remmel, and Weber [3]). The coding is a labeling of the edges of $2^{\omega}$ (or equivalently, all nodes in $2^{<\omega}$ except $\epsilon$ ) by the digits of $x$. Having labeled the edges according to $x$, the function $F_{x}$ coded by $x$ is defined by $F_{x}(y)=z$ if $z$ is the element of $2^{\omega}$ left over after following $y$ through the labeled tree and removing the 2 's. (In the case where only finitely many 0 's and 1 's remain after removing the 2 's, $F_{x}(y)$ is undefined.)

Journal of Logic \& Analysis 7:7 (2015) 
Formally, define a labeling function $\ell_{x}: 2^{<\omega} \backslash\{\epsilon\} \rightarrow 3$ by $\ell_{x}\left(\sigma_{i}\right)=x_{i-1}$ (recall that $\left(\sigma_{i}\right)_{i \in \omega}$ is the standard enumeration of $\left.2^{<\omega}\right)$. Now $F_{x} \in \mathcal{F}\left(2^{\omega}\right)$ is defined by $F_{x}(y)=z$ if and only if $z$ is the result of removing the 2 's from the sequence

$$
\ell_{x}(y \uparrow 1), \ell_{x}\left(y\lceil 2), \ell_{x}(y \uparrow 3), \ldots\right.
$$

Note that every $F \in \mathcal{F}\left(2^{\omega}\right)$ has infinitely many codes.

Definition 3.11 A function $F \in \mathcal{F}\left(2^{\omega}\right)$ is a random continuous function if $F=F_{x}$ for some $x \in \mathrm{MLR}_{\lambda}$.

Remark 3.12 $F_{x}$ is continuous (on its domain) because it is computable relative to some oracle, namely $x$. Since $2^{\omega}$ is compact and Hausdorff, it follows that $F_{x}$ is a closed map and hence that $\operatorname{ran}(F)$ is $\Pi_{1}^{0, F}$.

We will make use of the following facts about random continuous functions.

Theorem 3.13 ([3]) If $F \in \mathcal{F}\left(2^{\omega}\right)$ is random and $x \in 2^{\omega}$ is computable, then $F(x) \in 2^{\omega}$ is random.

Theorem 3.14 ([3]) If $F \in \mathcal{F}\left(2^{\omega}\right)$ is random, then $F$ is total.

\subsection{Algorithmically random measures on $2^{\omega}$}

Let $\mathscr{P}\left(2^{\omega}\right)$ be the space of probability measures on $2^{\omega}$. Given $x \in 2^{\omega}$, the $n$-th column $x_{n}$ of $x$ is defined by $x_{n}(k)=1$ if and only if $x(\langle n, k\rangle)=1$, where $\langle n, k\rangle$ is some fixed computable bijection between $\omega^{2}$ and $\omega$. We write $x=\oplus_{n \in \omega} x_{n}$. We define a map $\Psi: 2^{\omega} \rightarrow \mathscr{P}\left(2^{\omega}\right)$ that sends a real $x$ to the measure $\mu_{x}$ satisfying (i) $\mu_{x}(\epsilon)=1$ and (ii) $\mu_{x}\left(\sigma_{n} 0\right)=r_{n} \cdot \mu_{x}\left(\sigma_{n}\right)$, where $r_{n}$ is the real number corresponding to the $n$-th

column of $x$ (that is, $\left.r_{n}=\sum_{x_{n}(i)=1} 2^{-(i+1)}\right)$ and $\sigma_{n}$ is the $n$-th element in the standard enumeration of $2^{<\omega}$. Hereafter we will simply identify each $x_{n}$ with the corresponding $r_{n}$. This coding was first given in Culver [8].

Definition 3.15 A measure $\mu \in \mathscr{P}\left(2^{\omega}\right)$ is a random measure if $\mu=\mu_{x}$ for some $x \in \mathrm{MLR}_{\lambda}$.

Let $P$ be the pushforward measure on $\mathscr{P}\left(2^{\omega}\right)$ induced by $\lambda$ and $\Psi$. Then we have the following. 
Theorem 3.16 ([8]) Let $\nu \in \mathscr{P}\left(2^{\omega}\right)$. Then $\nu \in \mathrm{MLR}_{P}$ if and only if $\nu=\mu_{x}$ for some $x \in \mathrm{MLR}_{\lambda}$.

The support of a measure $\mu$ on $2^{\omega}$ is defined to be

$$
\operatorname{Supp}(\mu)=\left\{x \in 2^{\omega}:(\forall n)[\mu(x \mid n)>0]\right\} .
$$

It is not hard to see that $\operatorname{Supp}(\mu)=2^{\omega}$ for every random measure.

In [8], it was shown, among other results, that random measures are atomless (that is, $\mu(\{x\})=0$ for every $\left.x \in 2^{\omega}\right)$ and that the reals that are random with respect to some random measure are precisely the reals in $M L R_{\lambda}$.

\section{Applications of Randomness Preservation and No Ran- domness Ex Nihilo}

In this section, we demonstrate the usefulness of preservation of randomness and the no randomness ex nihilo principle in the study of algorithmically random objects such as closed sets, continuous functions, and so on.

As a warm-up, we provide a new, simpler proof of a known result from Barmpalias, Brodhead, Cenzer, Dashti, and Weber [2].

Theorem 4.1 Every random closed set contains an element of $M R_{\lambda}$ and every element of $\mathrm{MLR}_{\lambda}$ is contained in some random closed set.

Proof We define a computable map $\Phi: \mathcal{C}\left(2^{\omega}\right) \times 2^{\omega} \rightarrow 2^{\omega}$ that pushes forward the product measure $\lambda_{\mathcal{C}} \otimes \lambda$ to $\lambda$ and satisfies $\Phi(C, x) \in C$ for every $(C, x) \in \mathcal{C}\left(2^{\omega}\right) \times 2^{\omega}$. Having done this, preservation of randomness and no randomness ex nihilo imply that the image of a $\left(\lambda_{\mathfrak{e}} \otimes \lambda\right)$-random pair is $\lambda$-random and any $\lambda$-random is the image of some $\left(\lambda_{\mathcal{e}} \otimes \lambda\right)$-random pair. The result then follows because by van Lambalgen's theorem (Theorem 3.7), a pair $(C, x)$ is $\left(\lambda_{\mathcal{e}} \otimes \lambda\right)$-random if and only if $C$ is $\lambda_{\mathcal{e}}$-random and $x$ is $\lambda$-random relative to $C$.

The map $\Phi$ provides a path through $C$ (when viewed as the paths through a tree) by using $x$ to tell us which way to go whenever we encounter a branching node. Specifically, having $\Phi(C, x) \uparrow n=\sigma$ such that $\llbracket \sigma \rrbracket \cap C \neq \emptyset$, we define $\Phi(C, x)(n)=0$ if $\llbracket \sigma 1 \rrbracket \cap C=\emptyset$ and $\Phi(C, x)(n)=1$ if $\llbracket \sigma 0 \rrbracket \cap C=\emptyset$. If neither $\llbracket \sigma 0 \rrbracket \cap C=\emptyset$ nor $\llbracket \sigma 1 \rrbracket \cap C=\emptyset$, then we define $\Phi(C, x)(n)=x(n)$. 
The map $\Phi$ is clearly computable. It pushes $\lambda_{\mathfrak{e}} \otimes \lambda$ forward to $\lambda$ because if $\Phi$ has output $\sigma \in 2^{n}$ after $n$ steps, then $\Phi$ outputs a next bit of 0 if and only if either $\llbracket \sigma 1 \rrbracket \cap C=\emptyset$ or both $\llbracket \sigma 1 \rrbracket \cap C$ and $\llbracket \sigma 0 \rrbracket \cap C$ are non-empty and $x(n)=0$. The former happens with probability $\frac{1}{3}$, and the latter happens with probability $\frac{1}{6}=\frac{1}{3} \cdot \frac{1}{2}$ by independence. The proof is now complete since $\frac{1}{3}+\frac{1}{6}=\frac{1}{2}$.

Let $F \in \mathcal{F}\left(2^{\omega}\right)$. We define the zeros of $F$ to be $Z_{F}=\left\{x: F(x)=0^{\omega}\right\}$, which is clearly a closed subset of $2^{\omega}$. In Barmpalias, Brodhead, Cenzer, Remmel, and Weber [3], the following was shown.

Theorem 4.2 ([3]) Let $F \in \mathcal{F}\left(2^{\omega}\right)$ be random. Then $Z_{F}$ is a random closed set provided it is non-empty.

In [3] it was conjectured that the converse also holds, but this was left open. We show that this is the case. To do so, we provide a new proof of Theorem 4.2, from which the converse follows immediately. We also make use of an alternative characterization of random closed sets due to Diamondstone and Kjos-Hanssen [9].

Just as a binary tree with no dead ends is coded by a sequence in $3^{\omega}$ (see the paragraph preceding Definition 3.8), an arbitrary binary tree is coded by a sequence in $4^{\omega}$, except now a 3 at a node indicates that the tree is dead above that node. That is, given $x \in 4^{\omega}$, we define a tree $S_{x} \subseteq 2^{<\omega}$ inductively as follows. First $\epsilon$, the empty string, is included in $S_{x}$ by default. Now suppose that $\sigma \in S_{x}$ is the $(i+1)$-st extendible node in $S_{x}$. Then

- $\sigma 0 \in S_{x}$ and $\sigma 1 \notin S_{x}$ if $x(i)=0$;

- $\sigma 0 \notin S_{x}$ and $\sigma 1 \in S_{x}$ if $x(i)=1$;

- $\sigma 0 \in S_{x}$ and $\sigma 1 \in S_{x}$ if $x(i)=2$;

- $\sigma 0 \notin S_{x}$ and $\sigma 1 \notin S_{x}$ if $x(i)=3$.

This coding can be thought of as a labeling of the nodes of $2^{\omega}$ by the digits of $x$; a 0 at a node means that only the left branch is included, a 1 means that only the right branch is included, a 2 means that both branches are included, and a 3 means that neither branch is included. Note that every closed set except $2^{\omega}$ itself has infinitely many codes.

Let $\mu_{G W}$ be the measure on $4^{\omega}$ induced by setting, for each $\sigma \in 4^{<\omega}$,

$$
\mu_{G W}(\sigma 0 \mid \sigma)=\mu_{G W}(\sigma 1 \mid \sigma)=2 / 9, \mu_{G W}(\sigma 2 \mid \sigma)=4 / 9, \text { and } \mu_{G W}(\sigma 3 \mid \sigma)=1 / 9 .
$$

Via this coding we can also think of $\mu_{G W}$ as a measure on Tree, the space of binary trees. Then the probability of extending a string in a tree by only 0 is $2 / 9$, by only 1 is 
$2 / 9$, by both 0 and 1 is $4 / 9$, and by neither is $1 / 9$. We call a tree $T G W$-random if it has a random code, ie, if there is $x \in \mathrm{MLR}_{\mu_{G W}}$ such that $T=S_{x}$ (here $G W$ stands for Galton-Watson, since $G W$-trees are obtained by a Galton-Watson process).

Lemma 4.3 (Diamondstone and Kjos-Hanssen [9]) A closed set $C$ is random if and only if $C$ is the set of paths through an infinite $G W$-random tree.

By means of Lemma 4.3 we prove:

Theorem 4.4 (i) For every random $F \in \mathcal{F}\left(2^{\omega}\right), Z_{F}$ is a random closed set provided that it is non-empty.

(ii) For every random $C \in \mathcal{C}\left(2^{\omega}\right)$, there is some random $F \in \mathcal{F}\left(2^{\omega}\right)$ such that $C=Z_{F}$.

Proof We define a computable map $\Psi: \mathcal{F}\left(2^{\omega}\right) \rightarrow$ Tree that pushes forward $\lambda_{\mathcal{F}}$ to $\mu_{G W}$ such that the set of paths through $\Psi(F) \cap \operatorname{dom}(F)$ is exactly $Z_{F}$. Given our representation of functions as members of $3^{\omega}$ and binary trees as members of $4^{\omega}$, we are really defining a computable map $\widehat{\Psi}: 3^{\omega} \rightarrow 4^{\omega}$ that pushes forward $\lambda$ to $\mu_{G W}$.

Given $F \in \mathcal{F}\left(2^{\omega}\right)$, which we think of as a $\{0,1,2\}$-labeling of the edges of the full binary tree, we build the desired tree by declaring that $\sigma \in \Psi(F)$ if and only if the labels by $F$ of the edges of $\sigma$ consists only of 0 's and 2's. More formally, as in the paragraph preceding Definition 3.11, $F$ comes with a labeling function $\ell_{F}: 2^{<\omega} \backslash\{\epsilon\} \rightarrow 3$ defined by $\ell_{F}\left(\sigma_{i}\right)=j$ if and only if $x(i)=j$ where $x$ is the given code for $F$. So $\sigma \in \Psi(F)$ if and only if $\ell_{F}(\sigma \mid k) \in\{0,2\}^{<\omega}$ for every $0<k \leq|\sigma|$. Clearly this map is computable.

Now we show that the map $\Psi$ pushes $\lambda_{\mathcal{F}}$ forward to $\mu_{G W}$. Suppose $\sigma \in \Psi(F)$, which, as stated above, means that $\ell_{F}(\sigma \mid k) \in\{0,2\}^{<\omega}$ for every $0<k \leq|\sigma|$. Then

$$
\sigma 0 \in \Psi(F) \& \sigma 1 \notin \Psi(F) \Leftrightarrow \ell_{F}(\sigma 0) \in\{0,2\} \quad \& \quad \ell_{F}(\sigma 1)=1 .
$$

The right-hand side of the equivalence occurs with probability $(2 / 3)(1 / 3)=2 / 9$. Similarly,

$$
\sigma 0 \notin \Psi(F) \quad \& \quad \sigma 1 \in \Psi(F) \Leftrightarrow \ell_{F}(\sigma 0)=1 \quad \& \quad \ell_{F}(\sigma 1) \in\{0,2\},
$$

where this latter event also occurs with probability $2 / 9$. Next,

$$
\sigma 0 \in \Psi(F) \& \sigma 1 \in \Psi(F) \Leftrightarrow \ell_{F}(\sigma 0) \in\{0,2\} \quad \& \quad \ell_{F}(\sigma 1) \in\{0,2\},
$$

with the latter event occurring with probability $(2 / 3)(2 / 3)=4 / 9$. Lastly,

$$
\sigma 0 \notin \Psi(F) \& \sigma 1 \notin \Psi(F) \Leftrightarrow \ell_{F}(\sigma 0)=\ell_{F}(\sigma 1)=1,
$$


where the event on the right-hand side occurs with probability $(1 / 3)(1 / 3)=1 / 9$. Now by construction, it follows immediately that any path through the tree $\Psi(F)$ is a sequence $X$ such that either $F(X)=0^{\omega}$ (in the case that $\ell_{F}(X \mid n)=0$ for infinitely many $n$ ) or $F(X) \uparrow$ (in the case that $\ell_{F}(X\lceil n)=0$ for only finitely many $n$ ).

By preservation of randomness and no randomness ex nihilo, a tree is GW-random if and only if it is the image of some random continuous function $F$. The conclusion then follows by Lemma 4.3.

One consequence of Theorem 3.13 and Theorem 4.4(ii), not noted in Barmpalias, Brodhead, Cenzer, Remmel, and Weber [3], is that the composition of two random continuous functions need not be random.

Corollary 4.5 For every random $F \in \mathcal{F}\left(2^{\omega}\right)$, there is some random $G \in \mathcal{F}\left(2^{\omega}\right)$ such that $G \circ F$ is not random.

Proof By Theorem 3.13, there is some $R \in \mathrm{MLR}$ such that $F\left(0^{\omega}\right)=R$. By Theorem 4.1, there is some random $C \in \mathcal{C}\left(2^{\omega}\right)$ containing $R$. By Theorem 4.4(ii), there is a $G \in \mathcal{F}\left(2^{\omega}\right)$ such that $G^{-1}\left(\left\{0^{\omega}\right\}\right)=C$.

It follows that $G\left(F\left(0^{\omega}\right)\right)=0^{\omega}$, which implies with Theorem 3.13 that $G \circ F$ is not random.

Another consequence of Theorem 4.4 is that we can answer another open question from [3] involving random pseudo-distance functions. Given a closed set $C \in \mathcal{C}\left(2^{\omega}\right)$, a function $\delta: 2^{\omega} \rightarrow 2^{\omega}$ is a pseudo-distance function for $C$ if $C$ is the set of zeros of $\delta$. In [3] it was shown that if $\delta$ is a random pseudo-distance function for some $C \in \mathcal{C}\left(2^{\omega}\right)$, then $C$ is a random closed set, but the converse was left open. By Theorem 4.4, the converse immediately follows.

Corollary 4.6 Let $C \in \mathcal{C}\left(2^{\omega}\right)$. $C$ has a random pseudo-distance function if and only if $C$ is a random closed set.

\section{The support of a random measure}

In the previous section, we established a correspondence between random closed sets and and random continuous functions: a closed set $C$ is random if and only if it is the 
set of zeros of some random continuous function. In this section, we establish similar correspondences between random closed sets and random measures.

Since the support of a measure $\mu$, ie, the set $\operatorname{Supp}(\mu)=\left\{x \in 2^{\omega}: \forall n \mu(x \mid n)>0\right\}$ is a closed set, one might hope to establish such a correspondence by considering the supports of random measures. However, it is not hard to see that for each random measure $\mu, \operatorname{Supp}(\mu)=2^{\omega}$.

If we consider a different computable measure on $\mathscr{P}\left(2^{\omega}\right)$ than the measure $P$ defined above in Section 3.4, then such a correspondence can be given. In the first place, we want a measure $Q$ on $\mathscr{P}\left(2^{\omega}\right)$ with the property that no $Q$-random measure has full support. In fact, we can choose a measure $Q$ such that each $Q$-random measure is supported on a random closed set.

Theorem 5.1 There is a computable measure $Q$ on $\mathscr{P}\left(2^{\omega}\right)$ such that

(i) every $Q$-random measure is supported on a random closed set, and

(ii) for every random closed set $C \subseteq 2^{\omega}$, there is a $Q$-random measure $\mu$ such that $\operatorname{Supp}(\mu)=C$.

Proof We will define the measure $Q$ so that each $Q$-random measure is obtained by restricting the Lebesgue measure to a random closed set. That is, each $Q$-random measure will be uniform on all of the branching nodes of its support.

We define $Q$ in terms of an almost total functional $\Phi: 3^{\omega} \rightarrow 2^{\omega}$. On input $x \in 3^{\omega}, \Phi$ will treat $x$ as the code for a closed set and will output the sequence $y=\oplus_{i \in \omega} y_{i}$ defined as follows. For each $i \in \omega$, we set

$$
y_{i}= \begin{cases}1^{\infty} & \text { if } x(i)=0 \\ 0^{\infty} & \text { if } x(i)=1 \\ 10^{\infty} & \text { if } x(i)=2\end{cases}
$$

If we think of the columns of $y$ as encoding the conditional probabilities of a measure $\mu_{y}$, then if $\left(\sigma_{i}\right)_{i \in \omega}$ is the standard enumeration of $2^{<\omega}$, these conditional probabilities are given by

$$
p_{\sigma_{i}}= \begin{cases}1 & \text { if } x(i)=0 \\ 0 & \text { if } x(i)=1 \\ 1 / 2 & \text { if } x(i)=2\end{cases}
$$

That is, $\Phi(x)=y$, where $y$ represents the unique measure $\mu_{y}$ such that $\mu_{y}(\sigma 0 \mid \sigma)=p_{\sigma}$ for each $\sigma \in 2^{<\omega}$. Let $Q$ be the measure on $\mathscr{P}\left(2^{\omega}\right)$ induced by the composition of $\Phi$ and the representation map $\Psi: 2^{\omega} \rightarrow \mathscr{P}\left(2^{\omega}\right)$ defined in Section 3.4. 
We now show that $\Phi$ maps each $x \in$ MLR to a $Q$-random measure supported on a random closed set. Let $x \in \mathrm{MLR}$ and set $\Phi(x)=y$. By preservation of randomness, $\Psi(\Phi(x))=\mu_{y}$ is $Q$-random.

Next, since $x \in \operatorname{MLR},\left[T_{x}\right]$ is a random closed set. We claim that $\operatorname{Supp}\left(\mu_{y}\right)=\left[T_{x}\right]$. Suppose that $\sigma \in 2^{<\omega}$ is the $(n+1)$-st extendible node of $T_{x}$. Then one of the following holds:

(a) $\sigma 0 \in T_{x}$ and $\sigma 1 \notin T_{x}$;

(b) $\sigma 0 \notin T_{x}$ and $\sigma 1 \in T_{x}$; or

(c) $\sigma 0 \in T_{x}$ and $\sigma 1 \in T_{x}$.

Moreover, we have

- Condition (a) holds iff $x(n)=0$ iff $\mu_{y}(\sigma 0 \mid \sigma)=1$ and $\mu_{y}(\sigma 1 \mid \sigma)=0$.

- Condition (b) holds iff $x(n)=1$ iff $\mu_{y}(\sigma 0 \mid \sigma)=0$ and $\mu_{y}(\sigma 1 \mid \sigma)=1$.

- Condition (c) holds iff $x(n)=2$ iff $\mu_{y}(\sigma 0 \mid \sigma)=\mu_{y}(\sigma 1 \mid \sigma)=1 / 2$.

One can readily verify that $\mu_{y}\left(\sigma^{\frown} i \mid \sigma\right)>0$ if and only if $\sigma^{\frown} i \in T_{x}$. Thus

$$
\begin{aligned}
Z \in \operatorname{Supp}\left(\mu_{y}\right) & \Leftrightarrow \mu_{y}(Z\lceil n)>0 \text { for every } n \\
& \Leftrightarrow \mu_{y}(Z\lceil(n+1) \mid Z\lceil n)>0 \text { for every } n \\
& \Leftrightarrow Z\left\lceil(n+1) \in T_{x} \text { for every } n\right. \\
& \Leftrightarrow Z \in\left[T_{x}\right] .
\end{aligned}
$$

We have thus established that $\mu_{\Phi(x)}$ is supported on the random closed set $\left[T_{x}\right]$.

Lastly, we verify that the conditions (i) and (ii) hold. For (i), given any $Q$-random measure $\nu$, by no randomness ex nihilo there is some $x \in \operatorname{MLR}$ such that $\nu=\mu_{\Phi(x)}$, which by the above discussion is supported on a random closed set. To show (ii), let $C \subseteq 2^{\omega}$ be a random closed set. By definition there is some Martin-Löf random $z \in 3^{\omega}$ such that $C=\left[T_{z}\right]$. Hence $\Psi(\Phi(z))$ is a $Q$-random measure $\nu$. By the above discussion, $\nu$ has support $\left[T_{z}\right]=C$, which establishes the claim.

Instead of changing the measure on $\mathscr{P}\left(2^{\omega}\right)$ we can also establish a correspondence between random closed sets and random measures by considering not the support of a random measure but what we refer to as its $1 / 3$-support.

Definition 5.2 Let $\mu \in \mathscr{P}\left(2^{\omega}\right)$ and set

$$
T_{\mu}=\{\sigma:(\forall i<|\sigma|)[\mu(\sigma\lceil(i+i) \mid \sigma\lceil i)>1 / 3]\} \cup\{\epsilon\} .
$$

Then the 1/3-support of the measure $\mu$ is the closed set $\left[T_{\mu}\right]$. 
Theorem 5.3 A closed set $C \in \mathcal{C}\left(2^{\omega}\right)$ is random if and only it is the 1/3-support of some random measure $\mu \in \mathscr{P}\left(2^{\omega}\right)$.

Proof We define an almost-total, computable, and Lebesgue-measure-preserving map $\Phi: 2^{\omega} \rightarrow 3^{\omega}$ that induces a map $\tilde{\Phi}: \mathscr{P}\left(2^{\omega}\right) \rightarrow \mathcal{C}\left(2^{\omega}\right)$ such that $\tilde{\Phi}(\mu)=\left[T_{\mu}\right]$. Given $x=\oplus x_{i} \in 2^{\omega}$ such that $\mu\left(\sigma_{i} \frown 0 \mid \sigma_{i}\right)=x_{i}$ for each $i$, then for $\sigma \in T_{\mu}$ (which must exist since $\epsilon \in T_{\mu}$ ),

- if $\mu(\sigma 0 \mid \sigma) \in[0,1 / 3)$, then $\sigma 1 \in T_{\mu}$ and $\sigma 0 \notin T_{\mu}$;

- if $\mu(\sigma 0 \mid \sigma) \in(2 / 3,1]$, then $\sigma 0 \in T_{\mu}$ and $\sigma 1 \notin T_{\mu}$;

- if $\mu(\sigma 0 \mid \sigma) \in(1 / 3,2 / 3)$, then $\sigma 0 \in T_{\mu}$ and $\sigma 1 \in T_{\mu}$; and

- if $\mu(\sigma 0 \mid \sigma)=1 / 3$ or $\mu(\sigma 0 \mid \sigma)=2 / 3$, then $\Phi(x)$ is undefined.

Clearly $\Phi$ is defined on a set of measure one since it is defined on all sequences $x$ such that $x_{i} \neq 1 / 3$ and $x_{i} \neq 2 / 3$ for each $i$. Observe that each $\sigma \in T_{\mu}$ extends to an infinite path in $\left[T_{\mu}\right]$. Thus, if $\sigma$ is the $(n+1)$-st extendible node in $T_{\mu}$, then the each of the events

- $\sigma 0 \in T_{\mu}$ and $\sigma 1 \notin T_{\mu}$,

- $\sigma 0 \notin T_{\mu}$ and $\sigma 1 \in T_{\mu}$, and

- $\sigma 0 \in T_{\mu}$ and $\sigma 1 \in T_{\mu}$,

occurs with probability $1 / 3$, as each of these events corresponds to the case that $\mu(\sigma 0 \mid \sigma) \in[0,1 / 3), \mu(\sigma 0 \mid \sigma) \in(2 / 3,1]$, or $\mu(\sigma 0 \mid \sigma) \in(1 / 3,2 / 3)$, respectively. It thus follows that the pushforward measure induced by $\lambda$ and $\Phi$ is the Lebesgue measure on $3^{\omega}$. By preservation of randomness, each random measure $\mu$ is mapped to a random closed set, and by no randomness ex nihilo, each random closed set is the image of a random measure under $\Phi$. This establishes the theorem.

\section{The range of a random continuous function}

In Barmpalias, Brodhead, Cenzer, Remmel, and Weber [3], it was shown that for each $y \in 2^{\omega}$,

$$
\lambda\left(\left\{x \in 2^{\omega}: y \in \operatorname{ran}\left(F_{x}\right)\right\}\right)=3 / 4 .
$$

from which it follows that every $y \in 2^{\omega}$ is in the range of some random $F \in \mathcal{F}\left(2^{\omega}\right)$. In this section, we prove that $\lambda(\operatorname{ran}(F)) \in(0,1)$ for every random function $F$. First we will prove that $\lambda(\operatorname{ran}(F))>0$ for each random function, from which it follows 
that no random function is injective, a fact previously shown by [3] using a different proof, and that the range of a random function is never a random closed set. This latter result improves result of [3] according to which the range of a random function is not necessarily a random closed set. Our proof that the measure of the range of a random function is positive requires us to prove some auxiliary facts about the measure induced by a random function.

To prove that $\lambda(\operatorname{ran}(F))<1$ for every $F \in \mathcal{F}\left(2^{\omega}\right)$, we will show that no random function is surjective, from which the result immediately follows. Our result on surjectivity also improves a result of [3] according to which not every random function is surjective.

We begin by proving the following, which is similar to a result in Culver [8] for random measures.

Lemma 6.1 Let $\lambda_{\mathcal{F}}$ be the measure on $\mathcal{F}\left(2^{\omega}\right)$ induced by the correspondence between $\mathcal{F}\left(2^{\omega}\right)$ and $3^{\omega}$. Then the measure $P_{\mathcal{F}}$ on $\mathscr{P}\left(2^{\omega}\right)$ induced by the map $F \mapsto \lambda \circ F^{-1}$ has barycenter $\lambda$; ie,

$$
\lambda(\sigma)=\int_{\mathscr{P}\left(2^{\omega}\right)} \mu(\sigma) d P_{\mathcal{F}}(\mu)
$$

for each $\sigma \in 2^{<\omega}$.

Proof By change of variables, it suffices to show that

$$
2^{-|\sigma|}=\int_{\mathcal{F}\left(2^{\omega}\right)} \lambda\left(F^{-1} \llbracket \sigma \rrbracket\right) d \lambda_{\mathcal{F}}
$$

for each $\sigma \in 2^{<\omega}$. Without loss of generality, we assume $\sigma=0^{n}$. We proceed then by induction on $n$.

Equation (1) holds when $\sigma=\epsilon$ since each random $F$ is total by Theorem 3.14.

Now supposing that equation (1) holds for $0^{n}$, we show it also holds for $0^{n+1}$. Suppose then that $\int_{\mathcal{F}\left(2^{\omega}\right)} \lambda\left(F^{-1} \llbracket 0^{n} \rrbracket\right) d \lambda_{\mathcal{F}}=2^{-n}$. To compute $\int_{\mathcal{F}\left(2^{\omega}\right)} \lambda\left(F^{-1} \llbracket 0^{n+1} \rrbracket\right) d \lambda_{\mathcal{F}}$, we note that by symmetry $\int_{\mathcal{F}\left(2^{\omega}\right)} \lambda\left(F^{-1} \llbracket 0^{n+1} \rrbracket\right) d \lambda_{\mathcal{F}}=2 \cdot \int_{\mathcal{F}\left(2^{\omega}\right)} \lambda\left(\llbracket 0 \rrbracket \cap F^{-1} \llbracket 0^{n+1} \rrbracket\right) d \lambda_{\mathcal{F}}$ and proceed to compute $s_{n+1}:=\int_{\mathcal{F}\left(2^{\omega}\right)} \lambda\left(\llbracket 0 \rrbracket \cap F^{-1} \llbracket 0^{n+1} \rrbracket\right) d \lambda_{\mathcal{F}}$.

Recall that any $F \in \mathcal{F}\left(2^{\omega}\right)$ can be viewed as a labeling by 0 's, 1 's, and 2's of the nodes of full binary branching tree (where the root node is unlabeled). We will compute $\int_{\mathcal{F}\left(2^{\omega}\right)} \lambda\left(\llbracket 0 \rrbracket \cap F^{-1} \llbracket 0^{n+1} \rrbracket\right) d \lambda_{\mathcal{F}}$, which is really the expected measure of $\llbracket 0 \rrbracket \cap F^{-1} \llbracket 0^{n+1} \rrbracket$, by considering the three equiprobable cases for the label of the node 0 for an arbitrary $F \in \mathcal{F}\left(2^{\omega}\right)$. The point is that a label 0 contributes to producing an output beginning with $0^{n+1}$ and then we only need to obtain $0^{n}$ to get $0^{n+1}$, the label 1 
rules out the possibility of producing an output beginning with $0^{n+1}$, and the label 2 neither contributes to nor rules out the possibility of producing an output beginning with $0^{n+1}$ and then we need to obtain $0^{n+1}$ above one of the nodes 00 or 01 to get $0^{n+1}$.

Case 1: If the node 0 is labeled with a 0 , then the measure of all sequences extending the node 0 that (after removing 2 's) yield an output extending $0^{n+1}$ is equal to $1 / 2$ times the measure of all sequences that yield an output extending $0^{n}$. Since what happens above the node 0 is independent of what happens at the node 0 , the expected measured of $F^{-1} \llbracket 0^{n+1} \rrbracket$ is, in this case, $1 / 2 \cdot s_{n}=1 / 2 \cdot 2^{-n}$.

Case 2: If the node 0 is labeled with a 1 , then the measure of all sequences extending the node 0 that (after removing 2 's) yield an output extending $0^{n+1}$ is equal to 0 .

Case 3: If the node 0 is labeled with a 2, then the measure of all sequences extending the node 0 that (after removing 2 's) yield an output extending $0^{n+1}$ is equal to $1 / 2$ times the measure of all sequences extending the node 0 that yield an output extending $0^{n+1}$. Since what happens above the node 0 is independent of what happens at the node 0 , the expected measured of $F^{-1} \llbracket 0^{n+1} \rrbracket$ is, in this case, $1 / 2 \cdot 2 s_{n+1}$, where the 2 coefficient of $s_{n+1}$ comes from the fact that the $0^{n+1}$ could be above the node 00 or 01 and the expected measure of $\llbracket 0 \rrbracket \cap F^{-1} \llbracket 0^{n+1} \rrbracket$ is the same as the expected measure of $\llbracket 1 \rrbracket \cap F^{-1} \llbracket 0^{n+1} \rrbracket$, by independence.

Putting this all together gives

$$
s_{n+1}=\frac{1}{3} \cdot \frac{1}{2} \cdot 2^{-n}+\frac{1}{3} \cdot 0+\frac{1}{3} \cdot \frac{1}{2} \cdot 2 s_{n+1}
$$

which yields $s_{n+1}=2^{-n} / 4$, as desired.

Lemma 6.2 (Hoyrup [11], relativized) Let $Q$ be a computable measure on $\mathscr{P}\left(2^{\omega}\right)$ with barycenter $\mu$. Then for any $z \in 2^{\omega}$,

$$
\mathrm{MLR}_{\mu}^{z}=\bigcup_{\nu \in \mathrm{MLR}_{Q}^{z}} \mathrm{MLR}_{\nu}^{z}
$$

Theorem 6.3 If $F \in \mathcal{F}\left(2^{\omega}\right)$ is random, then $\lambda(\operatorname{ran}(F))>0$.

Proof Fix a random $F \in \mathcal{F}\left(2^{\omega}\right)$. We show that $\operatorname{ran}(F)$ always contains an element of $\operatorname{MLR}_{\lambda}^{F}$. Since $\operatorname{ran}(F)$ is $\Pi_{1}^{0, F}$ by Remark 3.12, it follows by Proposition 3.2 that $\lambda(\operatorname{ran}(F))>0$. 
By preservation of randomness relative to $F$, if $x \in \mathrm{MLR}_{\lambda}^{F}$, then $F(x) \in \mathrm{MLR}_{\lambda \circ F^{-1}}^{F}$. But by Lemmas 6.1 and 6.2, $\operatorname{MLR}_{\lambda \circ F^{-1}}^{F} \subseteq \operatorname{MLR}_{\lambda}^{F}$, so $F(x) \in \operatorname{MLR}_{\lambda}^{F}$, as desired.

We have a new proof of the following result from Barmpalias, Brodhead, Cenzer, Remmel, and Weber [3].

Corollary 6.4 If $F \in \mathcal{F}\left(2^{\omega}\right)$ is random, then $F$ is not injective.

Proof For any $y \in 2^{\omega}$, a relativization of Theorem 4.4(i) shows that $F^{-1}(\{y\})$, if non-empty, is a random closed set relative to $y$ provided that $F$ is random relative to $y$. Since $\operatorname{ran}(F)$ has positive Lebesgue measure, there is some $y \in \operatorname{ran}(F)$ that is random relative to $F$ (and so clearly $F^{-1}(\{y\})$ is non-empty). Then by van Lambalgen's theorem, $F$ is also random relative to $y$. So $F^{-1}(\{y\})$ is a non-empty random closed set and hence has size continuum by Theorem 3.10. Thus $F$ is not injective.

Corollary 6.5 If $F \in \mathcal{F}\left(2^{\omega}\right)$ is random, then $\operatorname{ran}(F)$ is not a random closed set.

Proof By Theorem 3.9, every random closed set has Lebesgue measure 0. But by Theorem 6.3, the range of a random $F \in \mathcal{F}\left(2^{\omega}\right)$ has positive Lebesgue measure, and thus the conclusion follows.

From the proof of Corollary 6.4 we can also obtain the following.

Corollary 6.6 Let $F \in \mathcal{F}\left(2^{\omega}\right)$ be random. Then the measure $\lambda_{F}$ induced by $F$ is atomless, that is, $\lambda_{F}(\{x\})=0$ for every $x \in 2^{\omega}$.

Proof Let $F \in \mathcal{F}\left(2^{\omega}\right)$ be random and suppose that $z \in 2^{\omega}$ is an atom of $\lambda_{F}$, ie, $\lambda_{F}(\{z\})>0$. It follows that $z \in \operatorname{MLR}_{\lambda_{F}}^{F}$, since $z$ is not contained in any $\lambda_{F}$-nullsets. Moreover, since $\lambda_{F}(\{z\})>0, F^{-1}(\{z\})$ is non-empty. As we argued in the proof of Corollary $6.4, F^{-1}(\{z\})$ is a random closed set and thus has Lesbesgue measure zero by Theorem 3.9, contradicting our assumption.

We now turn to showing that $\lambda(\operatorname{ran}(F))<1$ for every random $F \in \mathcal{F}\left(2^{\omega}\right)$. Instead of proving this directly, we will first prove the following.

Theorem 6.7 If $F \in \mathcal{F}\left(2^{\omega}\right)$ is surjective, then $F$ is not random. 
To prove Theorem 6.7, we provide a careful analysis of the result from [3] stated at the beginning of this section, namely that for each $y \in 2^{\omega}$,

$$
\lambda\left(\left\{x \in 2^{\omega}: y \in \operatorname{ran}\left(F_{x}\right)\right\}\right)=3 / 4 .
$$

This result is obtained by showing that the strictly decreasing sequence $\left(q_{n}\right)_{n \in \omega}$ defined by

$$
q_{n}=\lambda\left(\left\{x \in 2^{\omega}: \operatorname{ran}\left(F_{x}\right) \cap \llbracket 0^{n} \rrbracket\right\}\right)
$$

converges to $3 / 4$ and using the fact that

$$
\lambda\left(\left\{x \in 2^{\omega}: \operatorname{ran}\left(F_{x}\right) \cap \llbracket 0^{n} \rrbracket\right\}\right)=\lambda\left(\left\{x \in 2^{\omega}: \operatorname{ran}\left(F_{x}\right) \cap \llbracket \sigma \rrbracket\right\}\right)
$$

for each $\sigma \in 2^{<\omega}$ of length $n$. This sequence $\left(q_{n}\right)_{n \in \omega}$ is obtained by using a case analysis to derive the following recursive formula:

$$
q_{n+1}=\frac{3}{2} \sqrt{1+4 q_{n}}-\frac{3}{2}-q_{n} .
$$

For details, see Barmpalias, Brodhead, Cenzer, Remmel, and Weber [3, Theorem 2.12].

For $F \in \mathcal{F}\left(2^{\omega}\right)$ and $\sigma \in 2^{<\omega}$, let us say that $F$ hits $\llbracket \sigma \rrbracket$ if $\operatorname{ran}(F) \cap \llbracket \sigma \rrbracket \neq \emptyset$. Thus, $q_{n}$ is the probability that a random $F \in \mathcal{F}\left(2^{\omega}\right)$ hits $\llbracket \sigma \rrbracket$ for some fixed $\sigma \in 2^{<\omega}$ such that $|\sigma|=n$.

We will proceed by proving a series of lemmas. First, for each $n \in \omega$, let $\epsilon_{n}$ satisfy $q_{n}=3 / 4+\epsilon_{n}$. Since

(i) $q_{n}>q_{n+1}$ for every $n$, and

(ii) $\lim _{n \rightarrow \infty} q_{n}=3 / 4$,

we know that each $\epsilon_{n}$ is non-negative and $\lim _{n \rightarrow \infty} \epsilon_{n}=0$. Moreover, we have the following.

Lemma 6.8 For each $n \geq 1$,
(a) $\epsilon_{n+1} \leq \frac{1}{2} \epsilon_{n}$,
(b) $\epsilon_{n} \leq 2^{-(n+2)}$,
(c) $\epsilon_{n+1} \geq \frac{1}{2} \epsilon_{n}-2^{-(2 n+5)}$, and
(d) $\epsilon_{n} \geq \frac{1}{2^{n+5}-1}$.

Proof First, let $n \geq 1$. If we substitute $3 / 4+\epsilon_{n+1}$ and $3 / 4+\epsilon_{n}$ for $q_{n+1}$ and $q_{n}$, respectively, into Equation (2), we obtain (after simplification)

$$
\epsilon_{n+1}=3 \sqrt{1+\epsilon_{n}}-3-\epsilon_{n} .
$$


Since $\sqrt{1+x} \leq 1+\frac{x}{2}$ on $[0,1]$, from (3) we can conclude

$$
\epsilon_{n+1} \leq 3\left(1+\frac{\epsilon_{n}}{2}\right)-3-\epsilon_{n}=\frac{1}{2} \epsilon_{n},
$$

thereby establishing (a). To show (b), we proceed by induction. Using the fact from [3] that $q_{1}=\frac{\sqrt{45}-5}{2}$, it follows by direct calculation that

$$
\epsilon_{1}=\frac{\sqrt{45}-5}{2}-\frac{3}{4} \leq 2^{-3}
$$

Next, assuming that $\epsilon_{n} \leq 2^{-(n+2)}$, it follows from (a) that

$$
\epsilon_{n+1} \leq \frac{1}{2} \epsilon_{n} \leq \frac{1}{2} 2^{-(n+2)}=2^{-(n+3)} .
$$

To show (c), for each fixed $n \geq 1$, we use a different approximation of $\sqrt{1+x}$ from below. By (b), since $\epsilon_{n} \leq 2^{-(n+2)}$, we use the Taylor series approximation $1+\frac{x}{2}$ of $\sqrt{1+x}$ centered at 0 on $\left[0,2^{-(n+2)}\right]$ with error term

$$
\max _{c \in\left[0,2^{-(n+2)}\right]} \frac{1}{4(1+c)^{3 / 2}} \frac{x^{2}}{2}=\frac{x^{2}}{8} .
$$

Thus,

$$
\sqrt{1+x} \geq 1+\frac{x}{2}-\left(2^{-(n+2)}\right)^{2} / 8=1+\frac{x}{2}-2^{-(2 n+7)}
$$

on $\left[0,2^{-(n+2)}\right]$. Combining this with Equation (3) yields

$$
\epsilon_{n+1} \geq 3\left(1+\frac{\epsilon_{n}}{2}-2^{-(2 n+7)}\right)-3-\epsilon_{n} \geq \frac{1}{2} \epsilon_{n}-2^{-(2 n+5)} .
$$

Lastly, to prove (d), first observe that

$$
\epsilon_{1}=\frac{\sqrt{45}-5}{2}-\frac{3}{4} \geq 2^{-4}
$$

and thus it certainly follows that

$$
\epsilon_{1} \geq \frac{1}{2^{6}-1} .
$$

Next, using (c), we verify by induction that for $n \geq 2$,

$$
\epsilon_{n} \geq \frac{1}{2^{n-1}} \epsilon_{1}-\left(2^{-(n+5)}+\ldots+2^{-(2 n+3)}\right) .
$$

For $n=2$, by part (c) we have

$$
\epsilon_{2} \geq \frac{1}{2} \epsilon_{1}-2^{-7}
$$

Supposing that

$$
\epsilon_{n} \geq \frac{1}{2^{n-1}} \epsilon_{1}-\left(2^{-(n+5)}+\ldots+2^{-(2 n+3)}\right),
$$


again by part (c) we have

$$
\begin{aligned}
\epsilon_{n+1} \geq \frac{1}{2} \epsilon_{n}-2^{-(2 n+5)} & \geq \frac{1}{2}\left(\frac{1}{2^{n-1}} \epsilon_{1}-\left(2^{-(n+5)}+\ldots+2^{-(2 n+3)}\right)\right)-2^{-(2 n+5)} \\
& =\frac{1}{2^{n}} \epsilon_{1}-\left(2^{-(n+6)}+\ldots+2^{-(2 n+4)}\right)-2^{-(2 n+5)} \\
& =\frac{1}{2^{n}} \epsilon_{1}-\left(2^{-(n+6)}+\ldots+2^{-(2 n+5)}\right),
\end{aligned}
$$

which establishes Equation (5). Combining Equations (4) and (5) yields

$$
\begin{aligned}
\epsilon_{n} & \geq \frac{1}{2^{n-1}} 2^{-4}-\left(2^{-(n+5)}+\ldots+2^{-(2 n+3)}\right) . \\
& =\frac{1}{2^{(n+3)}}-2^{-(n+4)}\left(2^{-1}+\ldots+2^{-(n-1)}\right) \\
& =\frac{1}{2^{(n+3)}}-2^{-(n+4)}\left(1-2^{-(n-1)}\right) \\
& \geq 2^{-(n+3)}-2^{-(n+4)} \\
& \geq 2^{-(n+4)} \\
& \geq \frac{1}{2^{n+5}-1} .
\end{aligned}
$$

Lemma 6.9 For $n \geq 1$, we have

$$
\frac{q_{n+1}}{q_{n}} \leq 1-2^{-(n+6)}
$$

Proof By Lemma 6.8(d),

$$
\epsilon_{n} \geq \frac{1}{2^{n+5}-1}=\frac{2^{-(n+5)}}{1-2^{-(n+5)}}
$$

which implies

$$
\left(1-2^{-(n+5)}\right) \epsilon_{n} \geq 2^{-(n+5)}=4 \cdot 2^{-(n+7)} \geq 3 \cdot 2^{-(n+7)} .
$$

Multiplying both sides by $1 / 2$ yields

$$
\frac{1}{2}\left(1-2^{-(n+5)}\right) \epsilon_{n} \geq \frac{3}{4} 2^{-(n+6)} .
$$

Expanding the left hand side and using the fact from Lemma 6.8(a) that $\frac{1}{2} \epsilon_{n} \geq \epsilon_{n+1}$, we have

$$
\frac{1}{2} \epsilon_{n}+\left(\frac{1}{4}+\ldots+2^{-(n+6)}\right) \epsilon_{n} \geq \frac{3}{4} 2^{-(n+6)}+\epsilon_{n+1},
$$

which is equivalent to

$$
\left(1-2^{-(n+6)}\right) \epsilon_{n}+\frac{3}{4}\left(1-2^{-(n+6)}\right) \geq \frac{3}{4}+\epsilon_{n+1} .
$$


This yields the inequality

$$
\left(1-2^{-(n+6)}\right) q_{n} \geq q_{n+1},
$$

from which the conclusion follows.

Lemma 6.10 For $n \geq 1$, we have

$$
\left(2\left(\frac{q_{n+1}}{q_{n}}\right)-1\right)^{2^{n}} \leq \frac{1}{\sqrt[32]{e}}<1 .
$$

Proof First, it follows from Lemma 6.9 that

$$
2\left(\frac{q_{n+1}}{q_{n}}\right)-1 \leq 1-2^{-(n+5)}
$$

and hence

$$
\left(2\left(\frac{q_{n+1}}{q_{n}}\right)-1\right)^{2^{n}} \leq\left(1-2^{-(n+5)}\right)^{2^{n}} .
$$

Next, it is straightforward to verify by cross-multiplication that

$$
\frac{2^{n+5}-1}{2^{n+5}} \leq \frac{2^{n+6}-1}{2^{n+6}}
$$

and

$$
\frac{2^{n+5}-1}{2^{n+5}} \leq\left(\frac{2^{n+6}-1}{2^{n+6}}\right)^{2}
$$

from which it follows that

$$
\left(\frac{2^{n+5}-1}{2^{n+5}}\right)^{2^{n}} \leq\left(\frac{2^{n+6}-1}{2^{n+6}}\right)^{2^{n+1}}
$$

Lastly, we have

$$
\lim _{n \rightarrow \infty}\left(1-2^{-(n+5)}\right)^{2^{n}}=\frac{1}{\sqrt[32]{e}}
$$

From Equation (6) and the fact that the sequence $\left(\left(1-2^{-(n+5)}\right)^{2^{n}}\right)_{n \in \omega}$ is non-decreasing and converges to $1 / \sqrt[32]{e}$, the claim immediately follows.

The proof of following result is essentially the proof of the effective Choquet capacity theorem in Brodhead, Cenzer, Toska, and Wyman [5]. We reproduce the proof here for the sake of completeness.

Lemma 6.11 The probability that a random continuous function $F$ hits both $\llbracket 0 \rrbracket$ and $\llbracket 1 \rrbracket$ is $2 q_{1}-1$, and the probability that $F$ hits both $\llbracket \sigma 0 \rrbracket$ and $\llbracket \sigma 1 \rrbracket$ for a fixed $\sigma \in 2^{<\omega}$ of length $n \geq 1$, given that $F$ hits $\llbracket \sigma \rrbracket$, is equal to $2\left(\frac{q_{n+1}}{q_{n}}\right)-1$. 
Proof First, let us write the probability that $F$ hits $\llbracket \sigma \rrbracket$ for some fixed $\sigma$ as $\mathbb{P}\left(F \in H_{\sigma}\right)$. Now since $\mathbb{P}\left(F \in H_{0}\right)=q_{1}$, it follows that $\mathbb{P}\left(F \in H_{1} \backslash H_{0}\right)=1-q_{1}$ (here we use the fact that every random function is total). By symmetry, $\mathbb{P}\left(F \in H_{0} \backslash H_{1}\right)=1-q_{1}$. Since $F$ is total with probability one, it follows that

$$
\mathbb{P}\left(F \in H_{0} \cap H_{1}\right)=1-\left(\mathbb{P}\left(F \in H_{0} \backslash H_{1}\right)+\mathbb{P}\left(F \in H_{1} \backslash H_{0}\right)\right)
$$

and thus

$$
\mathbb{P}\left(F \in H_{0} \cap H_{1}\right)=1-\left(\left(1-q_{1}\right)+\left(1-q_{1}\right)\right)=2 q_{1}-1 .
$$

Next, let $\sigma$ be a string of length $n \geq 1$ and let $i \in\{0,1\}$. Since $\mathbb{P}\left(F \in H_{\sigma}\right)=q_{n}$ and $\mathbb{P}\left(F \in H_{\sigma \frown i}\right)=q_{n+1}$ it follows that

$$
\mathbb{P}\left(F \in H_{\sigma \frown i} \mid F \in H_{\sigma}\right)=\frac{\mathbb{P}\left(F \in H_{\sigma \frown i} \& F \in H_{\sigma}\right)}{\mathbb{P}\left(F \in H_{\sigma}\right)}=\frac{\mathbb{P}\left(F \in H_{\sigma} \frown_{i}\right)}{\mathbb{P}\left(F \in H_{\sigma}\right)}=\frac{q_{n+1}}{q_{n}} .
$$

Consequently,

$$
\mathbb{P}\left(F \in H_{\sigma 1} \backslash H_{\sigma 0} \mid F \in H_{\sigma}\right)=\mathbb{P}\left(F \in H_{\sigma 0} \backslash H_{\sigma 1} \mid F \in H_{\sigma}\right)=1-\frac{q_{n+1}}{q_{n}} .
$$

Thus,

$$
\begin{aligned}
\mathbb{P}\left(F \in H_{\sigma 0} \cap H_{\sigma 1} \mid F \in H_{\sigma}\right) & =1-\sum_{i=0}^{1} \mathbb{P}\left(F \in H_{\sigma \frown i} \backslash H_{\sigma \frown(i-1)} \mid F \in H_{\sigma}\right) \\
& =1-\left(\left(1-\frac{q_{n+1}}{q_{n}}\right)+\left(1-\frac{q_{n+1}}{q_{n}}\right)\right) \\
& =2\left(\frac{q_{n+1}}{q_{n}}\right)-1 .
\end{aligned}
$$

To complete the proof of Theorem 6.7, we now define a Martin-Löf test on $\mathcal{F}\left(2^{\omega}\right)$ that covers all surjective functions. Let us say that a function $F \in \mathcal{F}\left(2^{\omega}\right)$ is onto up to level $n$ if $F \in H_{\sigma}$ for every $\sigma \in 2^{n}$. By Lemmas 6.10 and 6.11, the probability of a function being onto up to level $n$ is

$$
\left(2 q_{1}-1\right) \prod_{i=1}^{n-1}\left(2\left(\frac{q_{i+1}}{q_{i}}\right)-1\right)^{2^{i}} \leq\left(\frac{1}{\sqrt[32]{e}}\right)^{n} .
$$

Thus, if we set

$$
U_{n}=\left\{F \in \mathcal{F}\left(2^{\omega}\right): F \text { is onto up to level } n\right\}
$$

and

$$
f(n)=\min \left\{k:(\sqrt[32]{e})^{-k} \leq 2^{-n}\right\},
$$

which is clearly computable, then $\left(U_{f(n)}\right)_{n \in \omega}$ is a Martin-Löf test with the property that $F \in \mathcal{F}\left(2^{\omega}\right)$ is onto if and only if $F \in \bigcap_{n \in \omega} U_{f(n)}$. This completes the proof. 
Corollary 6.12 If $F \in \mathcal{F}\left(2^{\omega}\right)$ is random, then $\lambda(\operatorname{ran}(F))<1$.

Proof Suppose $\lambda(\operatorname{ran}(F))=1$. Then since $\operatorname{ran}(F)$ is closed, it follows that $\operatorname{ran}(F)=2^{\omega}$. But then $F$ is onto, so it cannot be random.

We also have the following corollary.

Theorem 6.13 No measure induced by a random function is a random measure in the sense of Definition 3.15.

Proof Let $F \in \mathcal{F}\left(2^{\omega}\right)$ be random. Then by Corollary $6.12, \lambda(\operatorname{ran}(F))<1$. Thus, it follows that $2^{\omega} \backslash \operatorname{ran}(F)$ is non-empty and open, so $\llbracket \sigma \rrbracket \subseteq 2^{\omega} \backslash \operatorname{ran}(F)$ for some $\sigma \in 2^{<\omega}$. Thus, $\lambda_{F}(\sigma)=0$. By contrast, for every random measure $\mu$, we have $\mu(\sigma)>0$, and the result follows.

\section{Acknowledgements}

Porter was supported by the National Science Foundation under grant OISE-1159158 as part of the International Research Fellowship Program. Porter was also funded by the John Templeton Foundation as part of the project, "Structure and Randomness in the Theory of Computation." The opinions expressed in this publication are those of the authors and do not necessarily reflect the views of the John Templeton Foundation. Culver would also like to thank the John Templeton Foundation for supporting his visit to the University of Florida in March 2015. Lastly, the authors would like to thank an anonymous referee for helpful comments on an earlier draft of this paper.

\section{References}

[1] L M Axon, Algorithmically random closed sets and probability, $\mathrm{PhD}$ thesis, University of Notre Dame (2010)

[2] G Barmpalias, P Brodhead, D Cenzer, S Dashti, R Weber, Algorithmic randomness of closed sets, J. Logic Comput. 17 (2007) 1041-1062

[3] G Barmpalias, P Brodhead, D Cenzer, J B Remmel, R Weber, Algorithmic randomness of continuous functions, Arch. Math. Logic 46 (2008) 533-546

[4] L Bienvenu, C Porter, Strong reductions in effective randomness, Theoret. Comput. Sci. 459 (2012) 55-68 
[5] P Brodhead, D Cenzer, F Toska, S Wyman, Algorithmic randomness and capacity of closed sets, Log. Methods Comput. Sci. 7 (2011) 1-16

[6] D Cenzer, J B Remmel, Effectively closed sets (2014) Book manuscript

[7] D Cenzer, $\mathbf{R}$ Weber, Effective randomness of unions and intersections, Theory Comput. Syst. 52 (2013) 48-64

[8] Q Culver, Topics in Algorithmic Randomness and Effective Probability, $\mathrm{PhD}$ thesis, University of Notre Dame (2015)

[9] D Diamondstone, B Kjos-Hanssen, Martin-Löf randomness and Galton-Watson processes, Ann. Pure Appl. Logic 163 (2012) 519-529

[10] R Downey, D Hirschfeldt, Algorithmic randomness and complexity, Theory and Applications of Computability, Springer (2010)

[11] M Hoyrup, Computability of the ergodic decomposition, Ann. Pure Appl. Logic 164 (2013) 542-549

[12] A Nies, Computability and randomness, Oxford Logic Guides, Oxford University Press (2009)

[13] A Shen, One more definition of random sequence with respect to computable measure, from: "First World Congress of the Bernoulli Society on Math. Statistics and Probability theory, Tashkent" (1986)

[14] R I Soare, Recursively enumerable sets and degrees, Perspectives in Mathematical Logic, Springer-Verlag, Berlin (1987)

[15] M Van Lambalgen, The axiomatization of randomness, The Journal of Symbolic Logic 55 (1990) 1143-1167

[16] A K Zvonkin, L A Levin, The complexity of finite objects and the development of the concepts of information and randomness by means of the theory of algorithms, Russian Mathematical Surveys 25 (1970) 83

Department of Mathematics, Fordham University 441 E. Fordham Rd. Bronx, NY 10458

Department of Mathematics, University of Florida 1400 Stadium Rd. Gainesville, FL 32611

qculver@fordham.edu, cp@cpporter.com

Received: 10 March 2015 Revised: 19 January 2016 